\title{
Calidad de vida y pobreza en la Argentina (2010). Aproximación a escala provincial.
}

\section{Juan Pablo Celemín y Guillermo Ángel Velázquez}

CONICET

Tipo de trabajo: Artículo

Material original autorizado para su primera publicación en el Journal de Ciencias Sociales, Revista Académica de la Facultad de Ciencias Sociales de la Universidad de Palermo.

Recibido: 23-3-2015

Aceptado: 1-4-2015

\section{Resumen}

El estudio de la pobreza y de la calidad de vida posee elementos en común insoslayables, pero mientras que la pobreza es una medida de carencia que incluye a quienes no llegan a alcanzar un umbral mínimo establecido, la calidad de vida, en cambio, es una medida de logro respecto de un nivel establecido como óptimo. A partir de la elaboración de un índice de calidad de vida, el presente trabajo busca establecer el grado de asociación que tiene con el tradicional indicador de Necesidades Básicas Insatisfechas a escala provincial. El resultado indica una correlación de r=0,702 que deja margen a la discusión acerca de los alcances y limitaciones de ambas medidas. Mientras que el índice de calidad de vida puede ser modificado y adaptado a distintas realidades a la vez, el de Necesidades Básicas Insatisfechas presenta una estructura más limitada y rígida pero que, a su vez, facilita la comparación temporal. Además la representación cartográfica de ambos índices también registra diferencias territoriales. No obstante, para alcanzar resultados más definitivos es necesario complementar con estudios a escala departamental para minimizar los efectos de la agregación territorial.

Palabras clave: calidad de vida, necesidades básicas insatisfechas, correlación, provincias

\section{Abstract}

The study of poverty and quality of life has common elements but while poverty is a measure of lack

\footnotetext{
${ }^{1}$ Juan Pablo Celemín. Investigador Asistente CONICET. CONICET-UNCPBA (IGHCS). nimelec@yahoo.com.ar // Guillermo Ángel Velázquez. Investigador Superior del CONICET. Vicedirector IGHCS. CONICET-UNCPBA (IGHCS). guillevelaz@gmail.com
} 
that includes those who do not reach a minimum threshold, quality of life is a measure of achievement to an optimal level. From the development of a quality of life index, this paper seeks to establish its degree of association with the traditional indicator of unmet basic needs at the provincial scale. The results indicate a correlation of $r=0.702$ which leaves room for discussion about the scope and limitations of both. While the index of quality of life that can be modified and adapted to different realities, the unsatisfied basic needs indicator has a more limited and rigid structure that facilitates temporal comparison. Also the mapping of both indexes also showed territorial differences. However, to achieve more definitive results, more studies are needed at departmental level to avoid the effects of aggregation present at the provincial scale.

Keywords: quality of life, unsatisfied basic needs, correlation, provinces

\section{Introducción}

El término calidad de vida es ampliamente utilizado tanto en el lenguaje cotidiano como en distintas disciplinas científicas. Sin embargo, es importante remarcar que son múltiples las conceptualizaciones propuestas para detallarlo, y una de las principales dificultades reside en el hecho de que se incrementan en forma paralela a su uso y complejidad. Cuando se trabaja con un concepto difuso es muy difícil esbozar una definición única porque no hay acuerdo sobre cuál debería ser. Suele aparecer en distintos tipos de discursos, tanto científicos como periodísticos o políticos, y ha pasado a ser utilizado de forma casi masiva e ingenua, desconociendo la complejidad que lo caracteriza. La variedad de componentes que concentra y la imposibilidad de atender a todos, conduce necesariamente a la selección de ciertos aspectos. Con su uso suelen aparecer algunas nociones que en ocasiones se asimilan al concepto de calidad de vida, por lo que es necesario diferenciarlas ya que por su naturaleza conceptual, teórica y metodológica resultan substancialmente diferentes (Velázquez, 2005). Un primer paso para entenderla es distinguirla de conceptos similares. Tal es el caso del nivel de vida, condición de vida, bienestar o pobreza.

En general, se puede afirmar que la calidad de vida comprende, en primer término, la base material en la cual se desarrolla la vida; en segundo lugar, el ambiente natural y construido en el cual se desenvuelve el ser humano; y en última instancia, a todas las relaciones que devienen de las actividades realizadas, tanto el trabajo como otro tipo de relaciones sociopolíticas y culturales. Consecuentemente, se trata de un concepto de carácter evaluativo. 
El término condición de vida, de carácter descriptivo, se refiere a los aspectos económicos en cuanto contempla al consumo dirigido a la satisfacción de necesidades. Desde una perspectiva económica, Alarcón (2001) indica que las condiciones de vida dependen de gran cantidad de factores. Existe un conjunto de necesidades básicas que hay que cubrir simplemente para garantizar la subsistencia; pero hay otro que surge con el proceso de desarrollo y que se convierte en necesidades indispensables para funcionar socialmente. En cada momento, el nivel de lo que podríamos llamar necesidades básicas depende del nivel de desarrollo alcanzado y de los usos y costumbres de cada sociedad en particular. También indica que a medida que aumenta la capacidad productiva de los países, el conjunto de necesidades básicas y la calidad de los bienes para satisfacerlas se acrecienta. Desde esta perspectiva la autora considera a las condiciones de vida en asociación a la idea de consumo como elemento necesario para participar en la sociedad.

El nivel de vida de los individuos, conocimiento procedente de la economía, se refiere a los aspectos de naturaleza monetaria en cuanto contempla la idea de consumo de bienes y servicios, donde el poder adquisitivo se convierte en un mecanismo que permite lograr el desarrollo personal. El nivel de vida permite diferenciar un conjunto de individuos desposeídos de aquellos que cuentan con una serie de bienes materiales y capacidades para adquirirlos, diferenciando, en este sentido, a las clases sociales. Por su parte, Espinosa Henao (2000) indica que lo conocido como lujo y abundancia no necesariamente significa contar con lo óptimo en referencia a la calidad de vivir, ya que estos aspectos sólo proporcionan status social.

El concepto del bienestar, como apunta Camargo Mora (1999) presenta dos concepciones diferentes a lo largo del siglo XX. En un primer momento, se lo identifica desde una perspectiva principalmente cuantitativa asociada al conjunto de políticas y procesos económicos posteriores a la Segunda Guerra Mundial, conocido como Estado de Bienestar o Welfare. Así, el bienestar se asemeja a la idea de condición de vida y nivel de vida dado que su utilización está asociada con la implementación de políticas económicas y sociales que le imprimen mayor relevancia al consumo sostenido de bienes y servicios como motor de la economía. Más cercano en el tiempo, el significado de bienestar alcanza otra explicación más humanista (en sentido de well-being). Es a partir de los postulados de Amartya Sen (2001) sobre el desarrollo humano y calidad de vida donde se explica al bienestar en un sentido más amplio vinculado a las capacidades, oportunidades y ventajas de los individuos.

Si bien se reconoce el carácter multidimensional del concepto de pobreza en los estudios clásicos, su abordaje cuantitativo implica la selección de dimensiones e indicadores de bienestar relativos a 
condiciones materiales. El uso de indicadores se considera central para la formulación de políticas, programas y acciones puntuales así como la evaluación del alcance de los objetivos de gestión o impacto (Mathey, 2007).

Aunque los conceptos de "pobreza" y "calidad de vida" se refieren a fenómenos muy relacionados, tienen diferencias entre sí. La pobreza es una medida de carencia que incluye a quienes no llegan a alcanzar un umbral mínimo establecido. Estos umbrales pueden reflejar situaciones coyunturales (Línea de Pobreza-LP) o estructurales (índice de Población con Necesidades Básicas InsatisfechasNBI). Más recientemente, el INDEC ha elaborado una forma de medición de la pobreza "convergente" a través del Índice de Privación Material de los Hogares-IPMH.

La calidad de vida, en cambio, es una medida de logro respecto de un nivel establecido como óptimo, teniendo en cuenta dimensiones socioeconómicas y ambientales dependientes de la escala de valores prevaleciente en la sociedad, y que varían en función de las expectativas de progreso histórico (Velázquez, 2001).

Es decir que, mientras la pobreza se mide con respecto a un "piso", la calidad de vida se mide con respecto a un "techo". Mientras el piso de la pobreza es relativamente fijo, dado que apunta a la satisfacción de necesidades básicas, el techo de la calidad de vida es más variable (y ascendente), ya que la escala de valores y, sobre todo, las expectativas cambian.

Tampoco debemos confundir calidad de vida con nivel de vida, ya que esta última expresión se refiere habitualmente al nivel de consumo, es decir la adquisición de bienes y servicios -en muchos casos suntuarios-, pudiendo referir que el incremento en la posibilidad de consumo no implica necesariamente mejor calidad de vida.

En este marco conceptual, la finalidad del trabajo es responder el siguiente interrogante: ¿Qué correspondencia existe entre un Índice de Calidad de Vida (ICV), con su combinación de indicadores objetivos y subjetivos, y otro de pobreza como el de Necesidades Básicas insatisfechas? En esta primera aproximación al tema se trabaja a escala provincial y se recurre al coeficiente de correlación de Pearson para conocer la asociación entre ambos. Además se incluye cartografía de ambos índices para observar sus respectivas distribuciones espaciales.

\section{Metodología de un ICV y de NBI}

\subsection{Elaboración del ICV para la República Argentina}

Las fuentes para la medición de las diferencias de calidad de vida de la población argentina no son 
numerosas. Las más importantes de ellas son los Censos Nacionales, en el caso del presente trabajo son especialmente considerados los de noviembre de 2001 y octubre de 2010; y las Estadísticas Vitales del Ministerio de Salud y Acción Social de la Nación, ya que ambas cubren en su totalidad el territorio nacional, aunque con una disponibilidad de información inversa a la escala de análisis. En lo que respecta a los indicadores ambientales, ajustaremos para la escala provincial los índices de calidad ambiental propuestos en un trabajo anterior (Velázquez y Celemín, 2013).

Buena parte de las variables socioeconómicas referidas al conjunto del país no se encuentran disponibles a escala provincial (24 unidades), reduciéndose todavía más el conjunto para la escala departamental (511 unidades en el año 2001) o para niveles más detallados como los de fracción o radio censal (aproximadamente 5000 unidades en todo el país). Esta suerte de "paradoja de la información geográfica" implica que la información más interesante se encuentre disponible sólo para escalas poco interesantes; por consiguiente, y a pesar de los indudables progresos en el Sistema Estadístico Nacional, a medida que el nivel de análisis espacial se incrementa, la disponibilidad de información se "evapora".

Existe un problema metodológico adicional con respecto a la medición de los diferenciales de bienestar o calidad de vida de la población: el empleo de unidades territoriales relativamente "artificiales", tales como provincias y -aunque en menor medida- los departamentos/partidos, los cuales suelen no reflejar adecuadamente el grado de inequidad socio-territorial imperante. A este fenómeno, típico de la Geografía y de los Sistemas de Información Geográfica, se lo conoce como "Problema de la Unidad Espacial Modificable" (PUEM). De este modo, la división del territorio y los agrupamientos que se utilizan no son "neutros". Es posible, pues, encubrir o enmascarar desigualdades agrupando territorios y sociedades heterogéneos, pero ninguna división del territorio permite "crear" o "reproducir" inequidades, si estas no existen en la realidad.

En síntesis, pese a los mencionados avances del Sistema Estadístico Nacional y a las constantes innovaciones en el diseño metodológico y conceptual para la medición de las condiciones de vida de la población argentina, la formulación de un índice de bienestar con cierta pretensión de generalización o universalidad no es aún una cuestión totalmente resuelta, pues depende de numerosos factores tales como: procesos históricos, escala de valores de la sociedad, expectativas, vivencias individuales y colectivas, dimensiones privadas (ingresos, nivel de instrucción) y públicas (accesibilidad, cuestiones ambientales), escala de análisis, ajuste con la información disponible o geo-referenciación y validación de los resultados obtenidos. 
Para nuestro análisis hemos considerado dimensiones socioeconómicas (educación, salud y vivienda) y ambientales (recursos recreativos de base natural, recursos recreativos socialmente construidos y problemas ambientales). Estas dimensiones serán aproximadas a partir de la selección de variables y, posteriormente, traducidas a números-índice para garantizar su comparabilidad.

\subsubsection{Dimensiones para el estudio de la calidad de vida en la Argentina.}

La definición ajustada de un índice de calidad de vida "objetivo" no es una tarea sencilla ni universalmente válida. Basándonos en nuestras propias experiencias y en trabajos previos de investigación ampliamente discutidos en ámbitos específicos, ${ }^{2}$ hemos empleado dimensiones socioeconómicas (educación, salud, vivienda) y ambientales (problemas ambientales, recursos recreativos) para el dimensionamiento del bienestar de los argentinos.

A continuación explicaremos los indicadores seleccionados.

\section{Educación:}

- Porcentaje de población de 15 años o más que ya no asiste y con nivel de instrucción alcanzado menor a primario completo (cuadro 7.8 Censo 2001 y p 29 Censo 2010).

- Proporción de población de 15 años o más que ya no asiste y con nivel de instrucción alcanzado universitario completo (cuadros 7.8 Censo 2001 y p 29 Censo 2010).

\section{Salud:}

- Tasa de mortalidad infantil (TMI) según lugar de residencia de la madre (Ministerio de Salud. Dirección de Estadísticas e información de salud-DEIS).

- Proporción de población sin cobertura por obra social, plan de salud privado o mutual (cuadro 6.3 Censo 2001 y P 12 Censo 2010).

\section{Vivienda:}

- Porcentaje de población que reside en hogares que tienen inodoro sin descarga de agua o carecen de inodoro (cuadro 4.6 Censo 2001 y P 40 Censo 2010).

- Proporción de población en hogares hacinados, considerando como tales a aquellos que

2 Destacamos la Red de Investigadores Latinoamericanos de Calidad de Vida Urbana, las diversas sesiones sobre población y calidad de vida, realizadas en las Jornadas Argentinas de Estudios de Población (AEPA) y en los Seminarios Internacionales sobre Población y Sociedad en América Latina (SEPOSAL). 
superan las 2 personas por cuarto (cuadro 4.8 Censo 2001 y H 9 Censo 2010).

\section{Problemas ambientales y atracción del paisaje:}

La Argentina es un país caracterizado por gran diversidad de ambientes, singular riqueza en recursos

de base natural y de recursos recreativos socialmente construidos. Unos y otros pueden beneficiar o perjudicar las condiciones de vida de la población.

Dentro de estos ambientes se manifiestan problemas ambientales que inciden negativamente sobre la población. Entre ellos consideraremos:

- Uso de plaguicidas en agricultura

- $\quad$ Participación de Industria y minería en el PBG

- Contaminación/ Ruido /Congestionamiento

- $\quad$ Localizaciones peligrosas

- $\quad$ Localizaciones con Externalidades negativas

- Inseguridad (Tasa de hechos delictivos por cada 10.000 habitantes)

- $\quad$ Asentamientos precarios (\% de población residente en villas miseria)

- $\quad$ Basurales (\% de población residente a menos de 300 metros de un basural a cielo abierto)

- $\quad$ Sismicidad y vulcanismo

- Tornados

- Inundabilidad

- $\quad$ Confort climático

Como puede observarse en el listado de problemas considerados, estos deben ser concebidos en un sentido amplio dado que incluyen eventos de carácter estrictamente natural (tornados, inundaciones o sismos) y hechos vinculados a las acciones de la sociedad en recortes territoriales específicos.

También los recursos recreativos, ya sea de base natural o socialmente construidos, se comportan como factores de atracción que permiten mejorar la calidad de vida de la población, de allí que en la valoración del índice hemos considerado: 
a) Recursos recreativos de base natural

- Playas

- Balnearios a orillas de ríos, lagos, lagunas o diques

- Centros termales

- Nieve/hielo (posibilidad de actividades recreativas invernales)

- Relieve

- Espejos y cursos de agua

- Parques y espacios verdes

b) Recursos recreativos socialmente construidos

- Estética/Patrimonio urbano

- Centros culturales

- Centros comerciales y de esparcimiento

- Centros deportivos

Luego de esta enumeración de cada variable, explicaremos como utilizaremos esta información para construir un índice que abarque los aspectos que hemos considerado separadamente.

El primer paso para la elaboración del índice de calidad de vida es la transformación de las tasas en números-índice parciales, la que se realizó según el tipo de variable con el siguiente procedimiento:

a) Variables de costo (Problemas ambientales, Población con nivel de instrucción menor a primario, Población residente en hogares hacinados, Población sin obra social, Población sin retrete de uso exclusivo, viviendas deficientes y TMI).

$$
\mathrm{I}=\quad \text { Máximo }-\mathrm{a}
$$

\section{Máximo-mínimo}

b) Variables de beneficio (Población con nivel de instrucción universitario, Recursos recreativos de base natural y Recursos recreativos socialmente constituidos)

$$
\begin{aligned}
& \mathrm{I}=1-\quad \text { Máximo }-\mathrm{b} \\
& \text { Máximo-mínimo }
\end{aligned}
$$

Una vez transformadas las variables resulta posible la elaboración del índice de calidad de vida 
(tabla 1).

Tabla 1. Composición del índice de calidad de vida

\begin{tabular}{|l|l|l|l|l|}
\hline $\begin{array}{l}\text { Dimensión total } \\
\mathbf{( \% )}\end{array}$ & Indicadores & Variables & Peso parcial (\%) & Peso \\
\hline \multirow{4}{*}{$\begin{array}{c}\text { Socioeconómica } \\
\mathbf{6 0 \%}\end{array}$} & Educación & $\begin{array}{l}\text {-Ed<1ria } \\
\text {-Eduniversit }\end{array}$ & $\begin{array}{l}10 \\
10\end{array}$ & 20 \\
\cline { 2 - 5 } & Salud & $\begin{array}{l}\text {-TMl } \\
\text { - Sobsocial }\end{array}$ & 10 & 10 \\
\cline { 2 - 5 } & Vivienda & - Sretrete & 10 & 20 \\
\hline \multirow{2}{*}{$\begin{array}{l}\text { Ambiental } \\
\mathbf{4 0 \%}\end{array}$} & Índice de calidad & -RRBN & 10 & 20 \\
\hline & ambiental & -RRSC & 12 & \multirow{2}{*}{40} \\
\hline
\end{tabular}

Referencias: Ed<1ria: \% de población de 15 años y más sin instrucción o con nivel primario incompleto, Eduniversit: \% de población de 15 años y más con nivel de instrucción universitario completo. TMI: Tasa de mortalidad infantil, Sobsocial: \% de población sin obra social ni mutual, Sretrete: \% de población que reside en viviendas que carecen de inodoro de uso exclusivo o no posee descarga de agua, Hacinam: \% de población en hogares con más de 2 personas por cuarto, ICA: índice de calidad ambiental (Velázquez y Celemín, 2013), RRBN Recursos recreativos de base natural, RRSC Recursos recreativos socialmente construidos, PA: Problemas ambientales.

Como puede observarse en la Tabla 2 todos los indicadores considerados en el índice permiten afirmar que el complicado panorama que mostraba la calidad de vida de los argentinos en el 2001 se ha revertido en gran medida, aunque en forma asimétrica. Las condiciones socioeconómicas de la población argentina indudablemente mejoraron durante el último período intercensal.

Tabla 2: Evolución de indicadores socio-económicos en la Argentina. 1991-2010

\begin{tabular}{|l|l|l|l|l|l|l|}
\hline & $\begin{array}{l}\text { \% población } \\
\text { (ya no } \\
\text { asiste) }<1 \text { ria }\end{array}$ & $\begin{array}{l}\text { \% población } \\
\text { (ya no } \\
\text { asiste) } \\
\text { universitaria }\end{array}$ & TMI & $\begin{array}{l}\text { \% población } \\
\text { sin obra } \\
\text { social }\end{array}$ & $\begin{array}{l}\text { \% población } \\
\text { hacinada }\end{array}$ & $\begin{array}{l}\text { \% población } \\
\text { sin retrete }\end{array}$ \\
\hline 1991 & 22,08 & 3,81 & 21,7 & 37,76 & $27,20\left(^{*}\right)$ & 21,86 \\
\hline 2001 & 17,90 & 4,39 & 16,6 & 48,05 & 29,15 & 16,86 \\
\hline 2010 & 12,86 & 6,84 & 11,9 & 36,08 & 28,06 & 15,16 \\
\hline
\end{tabular}

Fuente: Censos nacionales y elaboración personal

(*) El dato de 1991 corresponde a \% de hogares 
Con respecto a los indicadores de educación, se ha registrado una disminución sustantiva de la escolaridad deficiente. Esto se vincula con el objetivo de incrementar la contención en el sistema educativo formal, probablemente en un contexto de fragmentación de los circuitos educativos. El otro extremo de la pirámide educativa, el de la población universitaria, aumentó significativamente su proporción. Durante este lapso, de la mano del incremento presupuestario a la educación superior, se crearon varias universidades en diferentes puntos del país, incrementando la oportunidad de acceso a algunos sectores de la población.

La situación de la salud también ha mejorado ostensiblemente. Más allá del proceso de transición demográfica, la Argentina ha disminuido su mortalidad infantil en términos generales $y$, como veremos, en forma sustantiva en algunas de las jurisdicciones más vulnerables. Por otra parte, la cobertura de salud también se ha incrementado fuertemente, logrando revertir los retrocesos verificados en el 2001. Este proceso se explica en parte por el incremento del presupuesto de salud y en parte por el desempeño del mercado laboral durante este lapso.

Los indicadores de vivienda son los que menos avances evidencian. Al deterioro de las viviendas existentes se suma la aún insuficiente construcción de nuevas unidades destinadas a sectores populares. El segmento más dinámico siguió constituyéndolo la demanda solvente, destinada a sectores minoritarios con altos ingresos.

\subsection{Necesidades Básicas Insatisfechas}

Las Necesidades Básicas Insatisfechas (NBI) constituyen un indicador válido para captar la pobreza estructural en determinada área. Comprenden el primer grupo de indicadores introducido por la Comisión Económica para América Latina (CEPAL) a comienzos de los años ochenta para identificar carencias críticas de la población y caracterizar la pobreza (Feres y Mancero, 2001: 8).

Se considera pobre a un hogar o a las personas que habitan en dicho hogar, cuando reúnen una o más de las siguientes condiciones: 1) Hacinamiento: hogares con más de tres personas por cuarto; 2) Vivienda: hogares que habitan en una vivienda de tipo inconveniente (pieza de inquilinato, pieza de hotel o pensión, casilla, local no construido para habitación o vivienda móvil, excluyendo casa, departamento y rancho); 3) Condiciones sanitarias: hogares que no tienen ningún tipo de retrete; 4) Asistencia escolar: hogares que tienen al menos un niño en edad escolar (6 a 12 años) que no asiste a la escuela; y 5) Capacidad de subsistencia: hogares que tienen cuatro o más personas dependientes por cada miembro de la familia ocupado, cuyo jefe no haya completado el tercer grado 
de escolaridad primaria.

\section{Resultados}

Una inicial diferenciación de los mapas de ICV y población sin NBI (Figuras 1 y 2) permite distinguir que la configuración espacial de la población sin NBI responde a la tradicional distribución socioeconómica presente en el país. Es decir que las provincias del norte muestran los porcentajes más altos de población sin NBI, a la vez que los más bajos se encuentran en el centro y en la Patagonia. Por su parte, el mapa del ICV muestra que en el norte del país se encuentran las provincias con peor desempeño (Chaco, Formosa y Santiago del Estero). Tucumán, Buenos Aires y Corrientes registran valores medio-bajos, mientras que el resto de las jurisdicciones se encuentran en valores medio-alto y altos.

Figura 1. Porcentaje de población sin NBI. Argentina 2010.

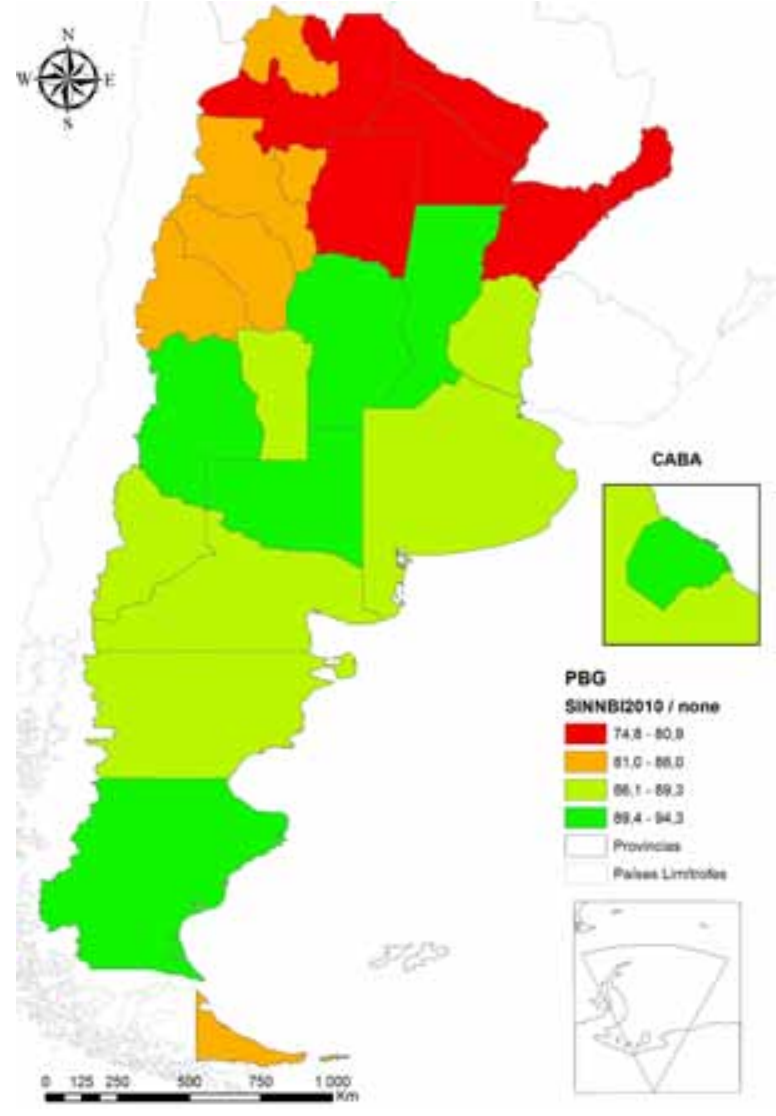

Fuente: elaboración personal 
Figura 2. Índice de calidad de vida. Argentina 2010.

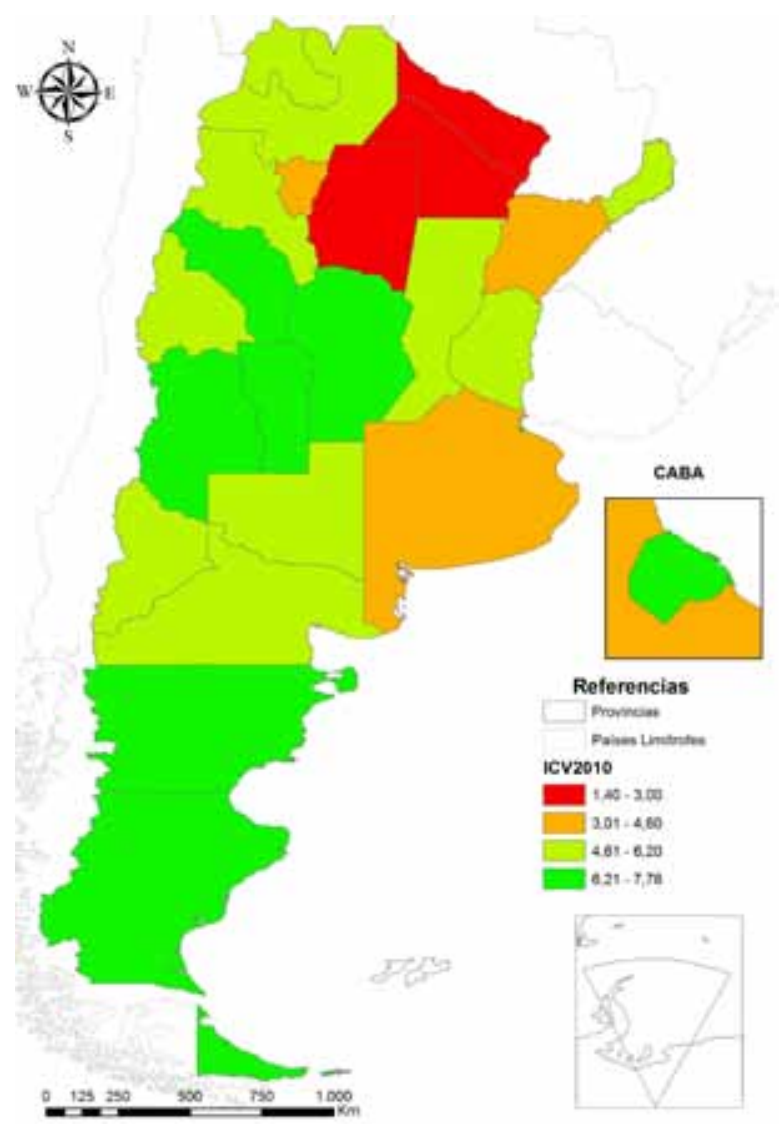

Fuente: elaboración personal

La descripción de los mapas se puede completar con el gráfico de dispersión (Figura 3) donde se observa con claridad como cada provincia se comporta en relación a ambos índices. En el extremo inferior izquierdo se encuentran las que presentan un bajo desempeño relativo en ambos índices (Chaco, Formosa, Santiago del Estero) mientras que en el extremo opuesto se encuentran las jurisdicciones con mejor situación relativa (Córdoba, Ciudad Autónoma de Buenos Aires). El resto de la mayoría de las provincias se encuentra en valores medios, siendo un caso particular la provincia de Buenos Aires con un ICV bajo en relación a población sin NBI. 
Figura 3. Relación ICV- población sin NBI. Argentina, 2010.

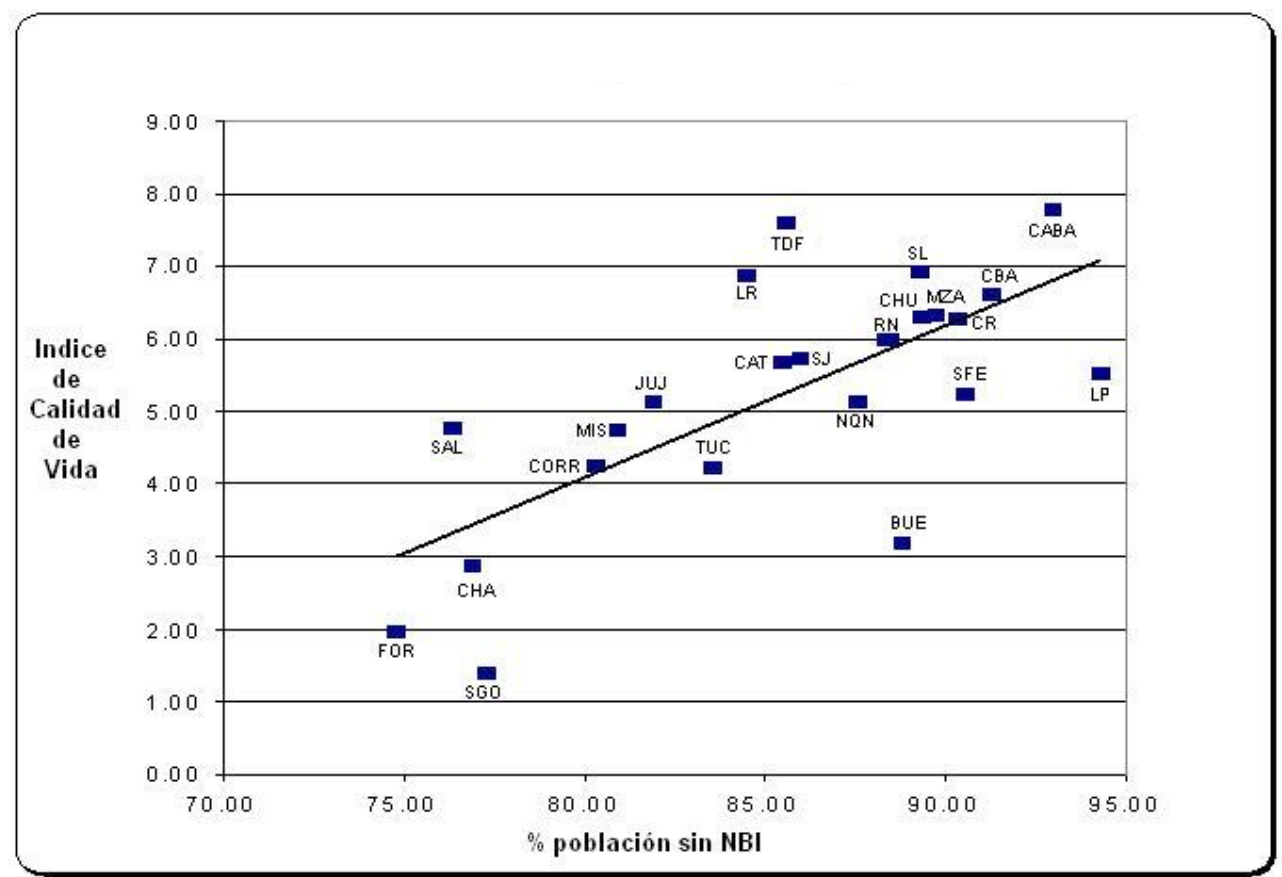

Fuente: elaboración personal

El principal resultado del trabajo es el coeficiente de correlación entre el ICV y población sin NBI que puede ser categorizado como alto al alcanzar el valor de $\mathbf{r = 0 , 7 0 2}$. Así observamos una visible correspondencia entre ambos índices, aunque existe un claro margen respecto a la correlación óptima (en teoría sería de 1) que permite explorar las diferencias constitutivas de ambos índices, en particular las dimensiones del ICV.

A futuro será necesario trabajar con mayor nivel de desagregación ambos índices de manera de paliar los efectos de agregación resultantes del Problema de la Unidad Espacial Modificable. Para ello deberá profundizarse la escala de análisis territorial con el propósito de indagar más detalladamente esta relación entre ICV y NBI. De esta manera se obtendrán datos más complejos para el análisis de la calidad de vida y de la pobreza en el país.

\section{Conclusiones}

La escala provincial permite, por ahora, una aproximación preliminar a la relación entre calidad de vida y pobreza en la Argentina. Se puede destacar inicialmente la utilidad de la información censal que, al ser integrada con otras fuentes de información complementarias, permite observar y cuantificar los éxitos y limitaciones de las políticas implementadas durante los últimos años, constituyéndose 
en herramienta fundamental en la planificación futura del país. Tradicionalmente, el NBI ha sido el indicador utilizado para medir la pobreza en el país, aunque otro tipo de índices más abarcativos pueden ser creados para enfocarse en la evolución de la calidad de vida. Tanto el ICV como el NBI presentan ventajas y desventajas en su elaboración y aplicación. EI ICV requiere de mucho más tiempo en su elaboración, sumado a la complejidad de trabajar tanto con indicadores subjetivos como objetivos. Además, las ponderaciones deben correr por cuenta de los investigadores. Entre sus aspectos positivos se destaca la amplia variedad de dimensiones y variables que utiliza, permitiendo tener una visión más completa de la calidad de vida. Tal es el caso de la dimensión ambiental, muchas veces obviada en este tipo de análisis. Por su parte, el NBI presenta una cantidad de variables más acotada, enfocada en el tema de la pobreza. Se destaca por ser un índice estandarizado internacionalmente y que es provisto por el INDEC sin necesidad de procesamiento previo.

Desde la disciplina geográfica, el presente trabajo constituye un primer avance con respecto al vínculo entre pobreza y calidad de vida para la población argentina sostenido sobre datos del censo 2010, aunque respaldado por años de investigación dedicados a analizar y sistematizar información extraída de diversas fuentes (censos, estadísticas vitales, estadísticas municipales) como así también de reflexión y ajuste de la metodología empleada. En tal sentido, la labor continúa y se complejiza en adelante con el cometido de trabajar otras escalas y, de esta manera, evidenciar con mayor claridad las dinámicas socio-espaciales presentes en el territorio nacional respecto de la calidad de vida de su población. 


\section{Referencias Bibliográficas:}

Alarcón, D. (2001). Medición de las condiciones de vida. Instituto Interamericano para el Desarrollo Social-INDES/BID. Recuperado el 5 abril de 2007. http://indes. iadb. org.

Camargo Mora, M. (1999). Calidad de Vida y Capacidades Humanas. Revista Geográfica Venezolana, $40(2), 247-258$.

Espinosa Henao, O. (2006): Enfoques, Teorías y Nuevos Rumbos del Concepto Calidad de Vida. Recuperado el 7 de marzo de 2006. http://naya.org.ar/congreso2000/ponencias/Oscar-Mauricioespinosa.htm

Feres, J.C. \& Mancero, X. El método de las Necesidades Básicas Insatisfechas (NBI) y sus aplicaciones en América Latina (2001). Santiago de Chile: CEPAL.

Mathey, D. (2007). Métodos e indicadores para la estimación de la pobreza rural en la Argentina. Documento de trabajo, 35, 1-32.

Sen, A. (2001): Desarrollo y libertad. Barcelona: Planeta.

Velázquez, G; Linares, S; Mikkelsen, C. \& Celemín, J.P. (2013, septiembre). Calidad de vida en la Argentina (2001 y 2010) una primera aproximación a escala provincial. Documento presentado en el V Congreso Nacional de Geografía de Universidades Públicas, Mendoza, Argentina.

Velázquez, G. \& Celemín, J.P (2013). La calidad ambiental en la Argentina. Análisis regional y departamental (c.2010). Tandil: Centro de Investigaciones Geográficas.

Velázquez, G. (2001). Geografía, Calidad de Vida y Fragmentación en la Argentina de los Noventa. Análisis Regional y Departamental utilizando SIG's. Tandil: Centro de Investigaciones Geográficas. Velázquez, G. (2005). Calidad de vida en la Argentina (1991 y 2001). Una aproximación a escala provincial. Tiempo y Espacio, 15, 29-41. 